\title{
Bond Geometry and Phase Transition Mechanism of H-Bonded Ferroelectrics
}

\author{
A. Bussmann-Holder \\ Max-Planck-Institut für Festkörperforschung, D-70506 Stuttgart, Germany \\ K. H. Michel \\ Departement Natuurkunde, Universiteit Antwerpen UIA, B-2610 Wilrijk, Belgium
}

(Received 6 October 1997)

\begin{abstract}
Taking into account the bilinear coupling between the tunneling protons and the displacements of the electron shells of the surrounding $\mathrm{PO}_{4}$ groups, we present a new model of hydrogen-bonded ferroelectrics. The model is an extension of both the tunneling model and the nonlinear polarizability model and includes the geometrical aspects of the hydrogen bond. It leads to a structural phase transition and describes the isotope effect due to the substitution $\mathrm{H} / \mathrm{D}$ in $\mathrm{KH}_{2} \mathrm{PO}_{4}$ and the pressure dependence. [S0031-9007(98)05455-6]

PACS numbers: 77.80.Bh, 61.50.Ks, 63.70. $+\mathrm{h}$
\end{abstract}

For a long time, the understanding of the structural phase transitions in hydrogen-bonded ferroelectric materials (for a review, see [1]) has been based on the quantum tunneling model introduced by Blinc [2,3]. In the disordered phase at high temperature, each hydrogen ion (proton) occupies with equal probability two equilibrium positions in a symmetric double well potential. Proton tunneling between these positions opposes localization. With decreasing temperature, the tunnel mode frequency decreases and its softening induces a structural phase transition with long range order in the proton positions. Within this model, the phase transition is driven by the direct proton-proton interaction. The experimentally observed isotope effect [in $\mathrm{KH}_{2} \mathrm{PO}_{4}(\mathrm{KDP})$, the transition temperature is $T_{c}=122 \mathrm{~K}$, while in $\mathrm{KD}_{2} \mathrm{PO}_{4}$ (DKDP), $\left.T_{c}=229 \mathrm{~K}\right]$ is attributed to a change in tunneling frequency caused by the mass change from $H$ to $D$ [2-4]. In the last ten years, high-resolution neutron diffraction work [5] has provided increasing evidence that this view of the structural phase transition has to be extended. It has been shown that structural changes in the geometry of the hydrogen bond which accompany the H/D substitution (the so-called Ubbelohde effect [6]) are closely connected with the microscopic mechanism of the phase transition. It is found that the distance $\delta$ between the two equilibrium positions of the proton increases upon deuteration. The diffraction results [7] lead to the conclusion that $\delta$ is the principal factor which determines $T_{c}$. Since the geometry of the hydrogen bond depends on the surrounding lattice configuration, it is necessary [8] to develop a theory which takes into account in a consistent way the coupled host-and-tunneling system. The coupling between the proton tunneling mode and the optical mode vibration of the $\left[\mathrm{K}-\mathrm{PO}_{4}\right]$ complex in KDP has been treated previously by Kobayashi [9]. However, the geometrical aspects of the hydrogen bond are not given special attention. The coupling of the optical lattice mode to the tunneling mode is only a relatively small effect, while the direct proton-proton interaction drives the phase transition [9]. A different approach to hydrogen-bonded ferroelectrics has been proposed within the oxygen ion polarizability model [10-12], but there, too, the geometry of the hydrogen bond is not taken into account.

In the following, we will present a new model for hydrogen-bonded ferroelectrics where the structural configuration of the proton is included. Although we refer to KDP-DKDP, the theory is also applicable to other systems like $\mathrm{C}_{4} \mathrm{H}_{2} \mathrm{O}_{4}$ (squaric acid) [13] and $\mathrm{SC}\left(\mathrm{NH}_{2}\right)_{2}$ (thiourea) [14]. The model is based on the interplay between the tunneling motion of the protons (deuterons), the polarizability changes of the neighboring $\mathrm{PO}_{4}$ groups, and the optical phonon mode of the $\left[\mathrm{K}-\mathrm{PO}_{4}\right]$ complexes. In order to have an analytically tractable problem, we restrict ourselves to a two dimensional model of KDP, which is shown in Fig. 1. Here, a formula unit consists of a $\mathrm{K}^{+}$ ion, ${\mathrm{a} \mathrm{PO}_{4}}^{-}$group, and one single proton [the situation is analogous to $\mathrm{PbHPO}_{4}$ (LDP), which is an experimental model system for the study of hydrogen ordering phase

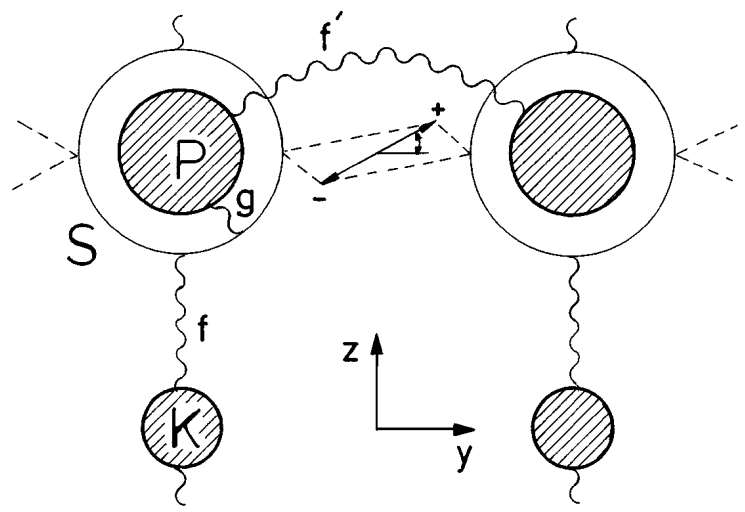

FIG. 1. Hydrogen bond geometry (not to scale): + and are $\mathrm{H}^{+}$positions $\delta / 2$ and $\delta / 2$, respectively; $P$ and $S$ are $\mathrm{PO}_{4}{ }^{--}$core and shell; $\mathrm{K}^{+}$is kalium ion; dotted lines indicate contributions to $C(\vec{\delta}) ; \Varangle$ is angle $\Psi$. 
transitions [7] ]. We treat the $\mathrm{PO}_{4}$ group as a composite structure which consists of a central core of mass $M_{P}$ surrounded by its electron shell of mass $m_{S}$, and neglect the tetrahedral shape of $\mathrm{PO}_{4}$. The displacement of the electron shell along the $z$ axis is an additional degree of freedom which accounts for the nonlinear polarizability of $\mathrm{PO}_{4}{ }^{--}$. The hydrogen bond is directed along the line which joins the centers of two neighboring $\mathrm{PO}_{4}$ groups, parallel to the $y$ axis. The geometry of the $\mathrm{H}$ positions in the paraelectric phase is inferred from diffraction experiments [5]. With respect to the center of the bond, the two equilibrium positions of the proton are $\left(\delta_{y} / 2, \delta_{z} / 2\right)$ and $\left(-\delta_{y} / 2,-\delta_{z} / 2\right)$. Here, $\delta_{y}=\delta \cos \Psi$ and $\delta_{z}=\delta \sin \Psi$, where $\Psi$ is the inclination angle between the $y$ axis and the proton hopping trajectory. In KDP, high-resolution neutron diffraction experiments yield [5] $\Psi=8.8^{\circ}$ and $\delta=0.3647 \AA$; in DKDP, $\Psi=7.4^{\circ}$ and $\delta=0.4462 \AA$. The bond symmetry is $\mathrm{C}_{2 h}$ [15]. The Hamiltonian

$$
H=H_{T}+H_{L}+H_{T L}
$$

consists of the tunneling protons $H_{T}$, the host lattice $H_{L}$, and a coupling $H_{T L}$. The tunneling model [2] of $N$ interacting protons is described by [16]

$$
H_{T}=-\frac{1}{2} \sum_{\vec{q}} J(\vec{q}) S^{z}(\vec{q}) S^{z}(-\vec{q})-\Omega S^{x}(\vec{q}=0) \sqrt{N}
$$

The equilibrium positions of each proton to the right or to the left of the bond center are described by the pseudospin $S^{z}$ with values +1 and -1 , respectively. In Fourier space $J(\vec{q})$ is the proton-proton interaction with wave vector $\vec{q}$. The operator $S^{x}$ accounts for proton tunneling; $\Omega$ is the tunnel frequency. The host lattice dynamics comprises displacements along the $z$ axis of the $\mathrm{K}^{+}$ions, of the $\mathrm{PO}_{4}$ cores, and of the $\mathrm{PO}_{4}$ shells. We write $H_{L}=T_{L}+U_{L}$, with potential energy

$$
\begin{aligned}
U_{L}= & \frac{1}{2} \sum_{\vec{q}}\left\{\left[\omega_{P}^{2}(\vec{q})+\frac{g}{M_{P}}\right] \phi_{P}^{\dagger}(\vec{q}) \phi_{P}(\vec{q})+\frac{2 f}{m_{K}} \phi_{K}^{\dagger}(\vec{q}) \phi_{K}(\vec{q})\right. \\
& \left.+(2 f+g) u^{\dagger}(\vec{q}) u(\vec{q})-2\left[\frac{2 f}{\sqrt{m_{K}}} \phi_{K}^{\dagger}(\vec{q})+\frac{g}{\sqrt{M_{P}}} \phi_{P}^{\dagger}(\vec{q})\right] u(\vec{q})\right\},
\end{aligned}
$$

and kinetic energy

$$
\begin{gathered}
T_{L}=\sum_{q} \frac{1}{2}\left\{\pi_{P}^{\dagger}(\vec{q}) \pi_{P}(\vec{q})+\pi_{K}^{\dagger}(\vec{q}) \pi_{K}(\vec{q})\right. \\
\left.+m_{S} \dot{u}^{\dagger}(q) \dot{u}(q)\right\} .
\end{gathered}
$$

Here, $\phi_{P} / \sqrt{M_{P}}$ denotes the displacement of the $\mathrm{PO}_{4}$ core center of mass, $\phi_{K} / \sqrt{m_{K}}$ is the displacement of the $\mathrm{K}^{+}$ ion, and $u$ and $\dot{u}$ are the displacement and velocity of the $\mathrm{PO}_{4}$ shell, respectively. The masses are $M_{P}, m_{K}$, and $m_{S}$, respectively; $\pi_{P}$ and $\pi_{K}$ are the momenta conjugate to $\phi_{P}$ and $\phi_{K}$. The Hamiltonian $H_{L}$ is equivalent to the one introduced for the nonlinear-polarizability model [12] of perovskites $\mathrm{ABO}_{3}$. The spring constant $f$ couples the motion of the $\mathrm{K}^{+}$ions to the $\mathrm{PO}_{4}$ shell displacements. The direct coupling $f^{\prime}$ between $\mathrm{PO}_{4}$ cores describes the transverse acoustic modes and is irrelevant in the long wavelength limit, where $\omega_{P}^{2}(\vec{q})$ vanishes. The local coreshell coupling $g$ consists of a harmonic attractive part $g_{2}$ and a fourth order repulsive anharmonic part $g_{4}$. In renormalized harmonic approximation, it reads

$$
\begin{aligned}
g= & g_{2}+\frac{3 g_{4}}{N} \\
& \times \sum_{\vec{q}}\left\langle\left(u^{\dagger}(\vec{q})-\frac{\phi_{P}^{\dagger}(\vec{q})}{\sqrt{M_{P}}}\right)\left(u(q)-\frac{\phi_{P}(\vec{q})}{\sqrt{M_{P}}}\right)\right\rangle .
\end{aligned}
$$

where the brackets \langle\rangle stand for a thermal average. The interaction $H_{T L}$ between the host lattice and the tunneling system is modeled by two back to back Morse potentials between each proton and the shells of the two neighboring $\mathrm{PO}_{4}{ }^{--}$groups [17]. The shells play, alternatively, the role of $\mathrm{H}^{+}$donor or acceptor. For each proton, the potential reads

$$
V=\sum_{\rho} \frac{\left(1+\epsilon_{\rho} S^{z}\right)}{2}\left\{V^{M}\left[\left|\vec{X}_{\rho}(r)\right|\right]+V^{M}\left[\left|\vec{X}_{\rho}(l)\right|\right]\right\} .
$$

Here, the index $\rho$ stands for \pm , with $\varepsilon_{+}=+1$ and $\varepsilon_{-}=$ -1 . The distance between the proton and the center of the $\mathrm{PO}_{4}$ shell to the right $(r)$ or to the left $(l)$ side of the bond center is $\left|\vec{X}_{ \pm}(\xi)\right|=\left\{\left(R-R_{0} \mp \varepsilon_{\xi} \delta_{y} / 2\right)^{2}+\right.$ $\left.\left[u(\xi) \mp \delta_{z} / 2\right]^{2}\right\}^{1 / 2}$, with $\varepsilon_{\xi}=+1$ or -1 for $\xi=r$ or $l$, respectively. The Morse potential reads

$$
V^{M}(|\vec{X}|)=D\left[e^{-2 \alpha|\vec{X}|}-2 e^{-\alpha|\vec{X}|}\right],
$$

where $2 R$ is the equilibrium distance between the centers of the two neighboring $\mathrm{PO}_{4}{ }^{-}$groups; $D, \alpha$, and $R_{0}$ are potential parameters. For the case of zero displacements in the $z$ direction, the potential $V$, Eq. (5), becomes a double minimum potential [17]. The experimental values [5] are $2 R=2.4946 \AA, \delta_{y}=0.3604 \AA$, and $\delta_{z}=$ $0.0558 \AA$ in KDP; and $2 R=2.5230 \AA, \delta_{y}=0.4424 \AA$, and $\delta_{z}=0.0575 \AA$ in DKDP. Expanding $V$ with respect to the shell displacements $u$ and summing over the lattice, we obtain, in the long wavelength regime,

$$
H_{T L}=C(\vec{\delta}) \sum_{\vec{q}} S^{\dagger z}(\vec{q}) u(\vec{q}),
$$

with

$$
\begin{aligned}
C(\vec{\delta})=-\frac{\delta_{z}}{2}[ & \frac{1}{\left|\vec{X}_{+}^{0}(r)\right|} \frac{\partial V^{M}\left[\left|\vec{X}_{+}^{0}(r)\right|\right]}{\partial\left|\vec{X}_{+}^{0}(r)\right|} \\
& \left.+\frac{1}{\left|\vec{X}_{-}^{0}(r)\right|} \frac{\partial V^{M}\left[\left|\vec{X}_{-}^{0}(r)\right|\right]}{\partial\left|\vec{X}_{-}^{0}(r)\right|}\right] .
\end{aligned}
$$


Here, the index 0 indicates that $\vec{X}_{ \pm}^{0}$ are taken at $u=0$. Although $C(\vec{\delta})$ changes sign under inversion of $\vec{\delta}$, physical quantities such as $T_{c}$ (see below) depend only on the absolute value of $C(\vec{\delta})$. The absolute values of $C(\vec{\delta})$ for KDP and DKDP are given in Table I. The interaction $H_{T L}$, which is inspired from the theory of translationrotation coupling in ionic molecular crystals [18], reflects the difference in electronic structure [15] between the donor and acceptor oxygen atoms.

We use standard Green's functions techniques [19] to describe the dynamics of protons and the displacements of ions. Starting from the Hamiltonian (1), we have obtained equations of motion for the retarded Green's functions $\left\langle\left\langle A^{\dagger}(\vec{q}) ; S^{z}(\vec{q})\right\rangle\right\rangle_{\omega}$, where the operator $A$ stands for $\phi_{P}, \phi_{K}, u$, or $S^{z}$ and $S^{y}$, and where $\omega$ is the external frequency. The $\mathrm{PO}_{4}$ shell displacements are eliminated by the adiabatic approximation. The coupled motion of the $\mathrm{K}$ and $\mathrm{PO}_{4}$ core masses is transformed to optical displacement coordinates:

$$
\xi(\vec{q})=\sqrt{\frac{\mu}{M_{P}}} \phi_{P}(\vec{q})-\sqrt{\frac{\mu}{m_{K}}} \phi_{K}(\vec{q}),
$$

TABLE I. Absolute values of coupling $C(\vec{\delta})$ (units $\mathrm{K} / \AA$ ) for several potential parameters $\alpha$ (units $\AA^{-1}$ ), and $R_{0}$ (units $\AA$ ). Values of $2 R, \delta_{x}$, and $\delta_{y}$ as given in the text; $\left.\delta\right|_{\mathrm{DKDP}} /\left.\delta\right|_{\mathrm{KDP}}=$ $1.22 ; D=34120 \mathrm{~K} ; r=\left.C\right|_{\mathrm{DKDP}} /\left.C\right|_{\mathrm{KDP}}$.

\begin{tabular}{cccc}
\hline \hline & $\left.C\right|_{\mathrm{KDP}}$ & $\left.C\right|_{\mathrm{DKDP}}$ & $r$ \\
\hline$\alpha=4.68$ & 27499.0 & 32193.9 & 1.171 \\
$R_{0}=1.00$ & & & \\
$\alpha=4.95$ & 29665.6 & 35036.1 & 1.181 \\
$R_{0}=1.00$ & & & \\
$\alpha=4.95$ & 20915.6 & 25209.56 & 1.201 \\
$R_{0}=0.95$ & & & \\
\hline \hline
\end{tabular}

where $\mu=m_{K} M_{P} /\left(m_{K}+M_{P}\right)$ is the reduced mass. Treating the tunneling system in molecular field approximation [3], we obtain, in the long wavelength limit,

$$
\begin{aligned}
\left(\omega^{2}-\omega_{0}^{2}\right)\left\langle\left\langle\xi^{\dagger}(\vec{q}) ; S^{z}(\vec{q})\right\rangle\right\rangle_{\omega}= & \frac{C \sqrt{\mu}}{(g+2 f)}\left[\frac{g}{M_{P}}-\frac{2 f}{m_{K}}\right] \\
& \times\left\langle\left\langle S^{z \dagger}(\vec{q}) ; S^{z}(\vec{q})\right\rangle\right\rangle_{\omega},
\end{aligned}
$$

$$
\left(\omega^{2}-\tilde{\Omega}^{2}\right)\left\langle\left\langle S^{z \dagger}(\vec{q}) ; S^{z}(\vec{q})\right\rangle\right\rangle_{\omega}=\left\langle S^{x}\right\rangle \Omega\left\{1+\frac{C \sqrt{\mu}}{(g+2 f)}\left[\frac{g}{M_{P}}-\frac{2 f}{m_{K}}\right]\left\langle\left\langle\xi^{\dagger}(\vec{q}) ; S^{z}(\vec{q})\right\rangle\right\rangle_{\omega}\right\} .
$$

Here, $\omega_{0}^{2}=2 f g / \mu(2 f+g)$ is the squared optical mode frequency of the host lattice, while $\tilde{\Omega}^{2}=\Omega^{2}-$ $\Omega\left\langle S^{x}\right\rangle\left[J+C^{2} /(g+2 f)\right]$, and $T$ is the temperature. The direct proton-proton interaction is given by $J \equiv J(\vec{q}=0)>0 . \quad$ In the following, we will always assume that $\Omega<T$ and, hence, $\left\langle S^{x}\right\rangle=\Omega / 4 T$. The renormalized tunneling frequency $\tilde{\Omega}$ is modified by the direct and by the $\mathrm{PO}_{4}$ shell mediated proton-proton interaction. By studying the resonances of the coupled system (10) and (11) in the static limit $\omega=0$, we find an instability under the condition

$$
\tilde{\Omega}^{2} \omega_{0}^{2}=\frac{C^{2} \Omega\left\langle S^{x}\right\rangle \mu}{(g+2 f)^{2}}\left(\frac{g}{M_{P}}-\frac{2 f}{m_{K}}\right)^{2},
$$

which corresponds to a structural phase transition of second order. Since $\left\langle S^{x}\right\rangle$ and $g$ depend on $T$, Eq. (12) determines the transition temperature $T_{c}$. In the absence of coupling $C$ between protons and $\mathrm{PO}_{4}$ motion, Eq. (12) reduces to $\tilde{\Omega}_{0}^{2} \omega_{0}^{2}=0$, where $\tilde{\Omega}_{0}^{2}=\Omega^{2}-\Omega\left\langle S^{x}\right\rangle J$ is the resonance frequency of the tunneling model, while $\omega_{0}^{2}$ is the resonance frequency of the polarizability model. A phase transition occurs either at (i) $\tilde{\Omega}_{0}^{2}=0$ (proton induced) or at (ii) $\omega_{0}^{2}=0$ (polarizability induced), depending on which instability takes place first with decreasing temperature.

In the presence of the coupling $C$, we first discuss analytically limiting cases. For a large optical mode frequency [both $f$ and $g$ are large, such that $J \gg C^{2} /(2 f+$ $g)$ ], the transition is driven by the condensation of the pro- ton motion. Relation (12) yields

$$
T_{c}=\frac{1}{4}\left\{J+\frac{C^{2}}{2 f+g}\left[1+\frac{\mu^{2}}{2 f g}\left(\frac{g}{M_{P}}-\frac{2 f}{m_{K}}\right)^{2}\right]\right\} .
$$

Here, we have assumed that the temperature dependence of $g$ is negligible. The coupling of the protons to the lattice tends to enhance $T_{c}$, but since $f$ and $g$ are large, this effect is small, in agreement with [9]. Oppositely, if the direct interaction between protons is negligible, $J=$ 0 , the phase transition is driven by the condensation of the optical mode. Then $g$ becomes small as a consequence of the nonlinear polarizability: $g<f ; \omega_{0}^{2} \approx g / \mu ; \tilde{\Omega}^{2} \approx$ $\Omega^{2}$. The $T$ dependence of the renormalized coupling $g$ is due to the second term on the right-hand side of Eq. (4). We eliminate the proton motion by means of Eq. (10) and write, for the mean square displacements of the optical mode,

$$
\frac{1}{N} \sum_{\vec{q}}\left\langle\xi^{\dagger}(\vec{q}) \xi(\vec{q})\right\rangle \approx T \chi_{0},
$$

where $\chi_{0}$ is the single particle susceptibility. Since the sum over $\vec{q}$ suppresses critical behavior, we approximate $\chi_{0}$ by a $T$-independent constant.

We then obtain

$$
g(T)=g_{2}+\frac{3 g_{4}}{\mu}\left[T+\frac{\mu}{m_{K}} \frac{C^{2}}{4 f}\right] \chi_{0},
$$




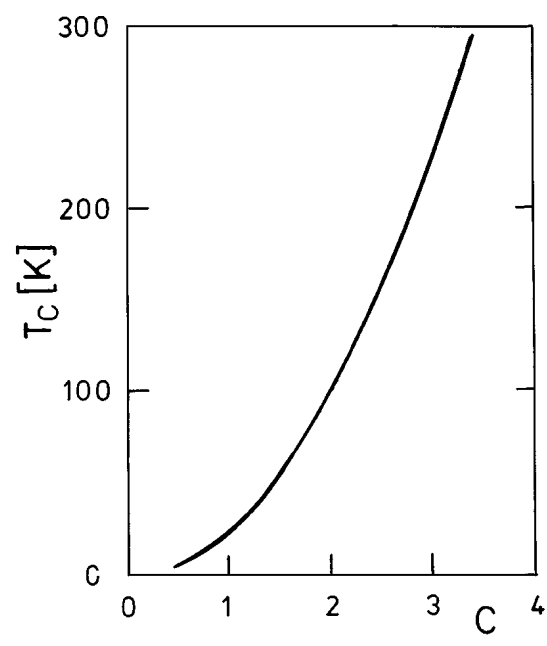

FIG. 2. Variation of $T_{c}$ with absolute value of coupling $C(\vec{\delta})$ (units $10^{4} \mathrm{~K} / \AA$ ).

and Eq. (12) reduces to

$$
g\left(T_{c}\right)-\left(\frac{\mu}{m_{K}}\right)^{2} \frac{C^{2}}{4 T_{c}}=0 .
$$

Here, $g\left(T_{c}\right)$ stands for the right-hand side of Eq. (15), with $T=T_{c}$. Defining $\hat{C}=\left(\mu / m_{K}\right)(C / 2)$, $\hat{g}_{4}=3 g_{4} \chi_{0} / \mu, \hat{f}=\left(\mu / m_{K}\right) f$, and $\theta=\left|g_{2}\right| / \hat{g}_{4}$, we solve Eq. (16) for $T_{c}>0$,

$$
T_{c}=\frac{\theta-\hat{C}^{2} / \hat{f}+\sqrt{\left(\theta-\hat{C}^{2} / \hat{f}\right)^{2}+4 \hat{C}^{2} / \hat{g}_{4}}}{2} .
$$

In the absence of the coupling $C$ between the proton and the $\mathrm{PO}_{4}$ shells, $T_{c}=\theta$, which is the transition temperature of the nonlinear polarizability model [10]. We recall that the coupling, Eq. (8), depends on the geometry of the hydrogen bond and, hence, expression (17) allows us to discuss the isotope effect. Assuming $\left(\hat{C}^{2} / \hat{f}\right)<\theta$ and $\left(4 \hat{C}^{2} / \hat{g}_{4}\right)^{1 / 2}<\theta$, we expand the righthand side of Eq. (17) in terms of $C$ and obtain $T_{c}=\theta+$ $\alpha \hat{C}^{2}$, where $\alpha=\left(\hat{f}-\left|g_{2}\right|\right) /\left(\left|g_{2}\right| \hat{f}\right)$. From the nonlinear polarizability model [10], we infer that $\hat{f}>\left|g_{2}\right|$ and, hence, $\alpha>0$. The coupling $C$ leads to an increase of $T_{c}$ and, since $\left.C\right|_{\mathrm{DKDP}}>\left.C\right|_{\mathrm{KDP}}$ (see Table I), the transition temperature is larger for DKDP than for KDP. We emphasize that the direct proton-proton interaction is negligible, while the interaction which is mediated by the coupling of the proton to the $\mathrm{PO}_{4}$ shells is essential.

We have solved numerically, Eq. (12), for $T_{c}$ as a function of the interaction parameters. We start from a set of parameters which yields $T_{c}$ for KDP close to the experimental value. For $C=21732 \mathrm{~K} / \AA$ and values of $g_{2}, g_{4}$, and $f$ close to the ones used for perovskite oxides [12], we get $\left.T_{c}\right|_{\mathrm{KDP}}=115 \mathrm{~K}$. In Fig. 2, we have plotted $T_{c}$ as a function of $C$, keeping the other parameters fixed. Deuteration affects $C$ and, from Table I, we have $r=\left.C\right|_{\mathrm{DKDP}} /\left.C\right|_{\mathrm{KDP}} \approx 1.2$. From Fig. 2 , we then find $\left.T_{c}\right|_{\text {DKDP }}=168 \mathrm{~K}$. We have checked that $C$ depends only weakly on $J, g_{2}$, and $g_{4}$, but strongly on $f$, the coupling between the $\mathrm{K}^{+}$ion and the $\mathrm{PO}_{4}$ shell. However, the last effect should not depend on deuteration.

Neutron diffraction experiments [5] have demonstrated the reduction of $\delta$ with pressure. Our results (Table I) indicate that $C$ decreases with decreasing $\delta$ and, from Fig. 2, we see that $T_{c}$ then decreases. Hence, the present theory accounts for the decrease of $T_{c}$ with increasing pressure [5,7].

We have presented a model for the phase transition mechanism in hydrogen-bonded ferroelectrics, which takes into account the effect of the $\mathrm{H}$ separation $\delta$ on the $\mathrm{PO}_{4}$ shell displacements. The theory describes, in a consistent way, two important phenomena: the increase of $T_{c}$ upon deuteration and the decrease of $T_{c}$ with applied pressure.

We acknowledge useful discussions with R. Blinc and N. Dalal. The work has been partially supported by the Fonds voor Wetenschappelijk Onderzoek, Vlaanderen.

[1] M. E. Lines and A. M. Glass, Principles and Applications of Ferroelectrics and Related Materials (Clarendon Press, Oxford, 1977).

[2] R. Blinc, J. Phys. Chem. Solids 13, 204 (1960).

[3] R. Blinc and B. Zeks, Adv. Phys. 21, 693 (1972).

[4] M. Tokunaga and T. Matsubara, Prog. Theor. Phys. 35, 857 (1966).

[5] J.E. Tibbals, R. J. Nelmes, and G. J. McIntyre, J. Phys. C 15, 37 (1982); Z. Tun, R. J. Nelmes, W.F. Kuhs, and R. D. F. Stansfield, J. Phys. C 21, 245 (1988).

[6] J. M. Robertson and A. R. Ubbelohde, Proc. R. Soc. London A 170, 222 (1939).

[7] M. I. McMahon, R. J. Nelmes, W.F. Kuhs, R. Dorwarth, R. O. Piltz, and Z. Tun, Nature (London) 348, 317 (1990).

[8] J. A. Krumhansl, Nature (London) 348, 285 (1990).

[9] K. K. Kobayashi, J. Phys. Soc. Jpn. 24, 497 (1968).

[10] A. Bussmann-Holder and H. Büttner, Phys. Rev. B 41, 9581 (1990).

[11] R. Migoni, H. Bilz, and D. Bäuerle, Phys. Rev. Lett. 37, 1155 (1976).

[12] A. Bussmann-Holder, H. Bilz, and G. Benedek, Phys. Rev. B 39, 9214 (1989).

[13] G. A. Samara and D. Semmigsen, J. Chem. Phys. 71, 1401 (1979).

[14] F. Denoyer, A. H. Moudden, R. Currat, C. Vettier, A. Bellamy, and M. Lambert, Phys. Rev. B 25, 1697 (1982).

[15] A. Katrusiak, Phys. Rev. B 51, 589 (1995); 48, 2992 (1993).

[16] P. G. de Gennes, Solid State Commun. 1, 132 (1963).

[17] M. C. Lawrence and G. N. Robertson, Ferroelectrics 25, 363 (1980); G. N. Robertson and M. C. Lawrence, J. Phys. C 14, 4559 (1981).

[18] K. H. Michel, Phys. Rev. B 24, 3998 (1981); R. M. Lynden-Bell and K.H. Michel, Rev. Mod. Phys. 66, 72 (1994).

[19] D. N. Zubarev, Sov. Phys. Usp. 3, 320 (1960). 